\title{
Safety Profile of Tolvaptan in the Treatment of Autosomal Dominant Polycystic Kidney Disease
}

\section{loannis Bellos (1D}

Laboratory of Experimental Surgery and Surgical Research N.S. Christeas, Athens University Medical School, National and Kapodistrian University of Athens, Athens, Greece
Correspondence: loannis Bellos Laboratory of Experimental Surgery and Surgical Research N.S. Christeas, Athens University Medical School, National and Kapodistrian University of Athens, 15B, Ag. Thoma str., Athens, II 2 27, Greece Tel +30694747044l

Email bellosg@windowslive.com

\begin{abstract}
Autosomal dominant polycystic kidney disease constitutes the most prevalent hereditary kidney disease, associated with high rates of morbidity leading eventually to endstage renal disease. Tolvaptan is a selective vasopressin antagonist and has emerged as a promising therapeutic option for patients with autosomal dominant polycystic kidney disease. The present review summarized current evidence regarding the safety profile of tolvaptan in patients with the disease. Consistent with its pharmacological action, aquaretic adverse events represent the most common side effects of tolvaptan, consisting of polyuria, pollakiuria and polydipsia. Gradual dose titration based on urinary osmolality, as well as dietary interventions aiming to reduce solute excretion, have been proposed as potential strategies to mitigate polyuria. In addition, tolvaptan administration may be complicated by liver injury, characterized by alanine aminotransferase and bilirubin elevations. Hepatotoxicity has been suggested to be triggered by impaired biliary clearance, activation of innate immunity and increased oxidative stress. Frequent monitoring of liver function tests has been shown to be effective in preventing Hy's Law and liver failure cases. Uric acid elevation due to reduced renal excretion may lead to hyperuricemia and gout, although no drug discontinuations have been linked to these events. Future studies should confirm the safety profile of tolvaptan in large-scale real-world studies, clarify the pathogenetic pathways leading to hepatotoxicity and define its role in special populations, especially pediatric patients.
\end{abstract}

Keywords: vaptan, ADPKD, adverse effect, toxicity, hepatotoxicity, liver

\section{Introduction}

Autosomal-dominant polycystic kidney disease (ADPKD) represents the most common hereditary renal disease, affecting a total of 12 million people worldwide. It constitutes the fourth leading cause of end-stage renal disease, with patients initiating dialysis during the sixth decade of life. ${ }^{1,2}$ Its etiology is based on mutations of PKD1 and PKD2 genes, which encode polycystin 1 and 2, respectively. Loss of polycystins from the renal primary cilia is associated with altered cell adhesion and dysregulated metabolic signaling, leading to the release of chemokines and pro-inflammatory cytokines which promote matrix remodeling and interstitial fibrosis. ${ }^{3}$ The renal phenotype of the disease includes the formation of fluid-filled cysts, abdominal or flank pain and concentration defects. In addition, ADPKD is linked to several extrarenal manifestations, especially liver cysts and intracranial aneurysms. ${ }^{4}$ Importantly, the majority of patients develop hypertension and endothelial dysfunction, ${ }^{5}$ leading to high rates of cardiovascular morbidity and mortality. ${ }^{6}$ 
Tolvaptan represents a non-peptide selective V2receptor antagonist. It is orally administered and exerts its pharmacologic action by inhibiting arginine vasopressin (AVP), leading to the downregulation of aquaporin-2 in the collecting duct and promoting aquaresis. ${ }^{7}$ The main indication of tolvaptan therapy is severe or resistant euvolemic and hypervolemic hyponatremia, especially in patients with syndrome of inappropriate antidiuretic hormone secretion (SIADH). ${ }^{8}$ Tolvaptan has been also proposed as a useful treatment option to improve fluid retention in the setting of acute heart failure ${ }^{9}$ or after cardiac surgery, ${ }^{10}$ limiting the required dose of loop diuretics. Furthermore, its administration has been shown to ameliorate congestive symptoms in patients with chronic kidney disease ${ }^{11}$ or refractory ascites, ${ }^{12}$ without increasing the risk of serious adverse effects. However, due to concerns of potential idiosyncratic liver toxicity, the US Food and Drug Administration (FDA) has issued a "black box warning" precluding the use of tolvaptan among patients with cirrhosis and impaired liver function. ${ }^{13}$

Increasing research interest exists regarding the role of tolvaptan in the management of ADPKD. The Tolvaptan Efficacy and Safety in Management of Autosomal Dominant Polycystic Kidney Disease and Its Outcomes (TEMPO) 3:4 randomized controlled trial ${ }^{14}$ initially indicated that tolvaptan administration in patients with preserved renal function was able to slow the increase in total kidney volume and the decline of renal function. Its openlabel extension TEMPO 4:4 trial ${ }^{15}$ confirmed the longterm decrease of the slope of glomerular filtration rate decline, although no persistent effects on total kidney volume were demonstrated. The beneficial effects of tolvaptan were also highlighted in ADPKD patients with impaired renal function since the Replicating Evidence of Preserved Renal Function: an Investigation of Tolvaptan Safety and Efficacy in ADPKD (REPRISE) trial ${ }^{16}$ showed that tolvaptan treatment was associated with attenuated decline of kidney function. As a result, the drug has been approved by the US Food and Drug Administration for the indication of slowing glomerular filtration rate drop in patients with progressive ADPKD. ${ }^{17}$

Concerns have been raised regarding the safety profile of tolvaptan, mainly due the risk of aquaresis-related adverse effects and hepatotoxicity. The aim of the present review is to summarize current evidence in the field and evaluate the frequency and severity of adverse events among patients receiving tolvaptan for the management of ADPKD.

\section{Materials and Methods}

The present study is a narrative review. Literature search was conducted by searching MEDLINE from inception using the following algorithm: "('Polycystic Kidney, Autosomal Dominant' [Mesh] OR ADPKD) AND (tolvaptan OR vaptan OR ((adh OR avp OR vasopressin) AND antagonist))". The date of the last search was 27 April 2021. Randomized controlled trials, cohort studies (both prospective and retrospective), pharmacovigilance studies, as well as case reports/series reporting the rate of tolvaptan-related adverse effects among patients with ADPKD were evaluated.

\section{Results \\ Mechanism of Action}

The central role of AVP in ADPKD has been suggested by animal studies indicating that V2-antagonism was strongly related to reduced renal cystogenesis. Specifically, attenuated activation of V2-receptors with gene mutations, increased water intake or AVP antagonists has been shown to lower cyst cell proliferation, limit fluid secretion and slow kidney enlargement. ${ }^{18,19}$ Conversely, the administration of the V2-agonist dDAVP (1-deamino-8-d-arginine vasopressin) was able to restore the cystic phenotype, increase kidney weight, promote fibrosis and aggravate renal function. ${ }^{20}$ Experimental evidence has proposed that inhibition of V2-activation with OPC-31260 leads to downregulation of renal cAMP (cyclic adenosine monophosphate) signaling, resulting in attenuated ERKmediated cell growth and reduced chloride-rich fluid secretion. ${ }^{21}$ As a result, AVP antagonism has been suggested to be sufficient to suppress the MEK/ERK signaling pathway and halt the progression of cyst expansion. ${ }^{22}$ In this context, tolvaptan, a derivative of OPC-31260, has been chosen as a candidate ADPKD treatment option, justifying its testing in human clinical trials. Interestingly, tolvaptan administration to ADPKD patients has been shown to decreased expression of semaphoring $7 \mathrm{~A}$ in T-lymphocytes, potentially reflecting reduced inflammation and renal fibrosis. ${ }^{23}$

\section{Aquaretic Adverse Effects}

Consistent with its pharmacodynamic properties, tolvaptan is commonly associated with aquaretic adverse effects, such as polyuria, pollakiuria, nocturia, thirst and dry mouth. Specifically, AVP antagonism in the collecting duct leads to decreased urine osmolality increased free 
water clearance and serum sodium levels. ${ }^{24}$ The TEMPO $3: 4$ trial $^{14}$ that compared to placebo, tolvaptan administration was linked with significantly higher frequency of thirst $(55.3 \%$ vs $20.5 \%)$, polyuria $(38.3 \%$ vs $17.2 \%)$, nocturia $(29.1 \%$ vs $13.0 \%)$, pollakiuria $(23.2 \%$ vs $5.4 \%)$ and polydipsia (10.4\% vs $3.5 \%$ ). Overall, $78 \%$ of the participants receiving tolvaptan reported at least one aquaretic adverse effect; of them, $10 \%$ discontinued the drug due to the aquaretic event. Patients that discontinued tolvaptan tended to be younger, with higher estimated glomerular filtration rate and higher baseline fasting urinary osmolality. ${ }^{25}$ Similarly, the TEMPO 4:4 extension trial ${ }^{15}$ results demonstrated that $9 \%$ of the patients that crossedover to tolvaptan therapy stopped the drug due to aquaresis. The REPRISE trial ${ }^{16}$ indicated that during its 5 -week single-blind period, polyuria was the most common adverse effect (31.7\%), while $4.6 \%$ of the patients discontinued tolvaptan due to aquaretic events. Torres et $\mathrm{al}^{26}$ reported the outcomes of a long-term open-label safety study including the participants of all randomized trials and indicated that polyuria and thirst were more prevalent among individuals with shorter duration of tolvaptan treatment, suggesting that aquaretic adverse effects may attenuate over time.

Gradual tolvaptan dose titration from 60 to 90 and eventually $120 \mathrm{mg}$ daily may decrease the risk of aquaretic adverse effects and improve tolerability. To this end, serial measurements urinary osmolality changes, aiming to achieve a target decrease of $\geq 300 \mathrm{mOsm} / \mathrm{kg}$, as well as to maintain an absolute urine osmolality below 300 $\mathrm{mOsm} / \mathrm{kg}$ may facilitate dose optimization. Alternatively, a reduction of at least $200 \mathrm{mOsm} / \mathrm{kg}$ may be targeted in early-stage patients with potentially increased propensity to aquaretic adverse effects. ${ }^{25}$ As in nephrogenic diabetes insipidus, solute excretion represents the main determinant of urine volume in tolvaptan-treated patients. ${ }^{27} \mathrm{As}$ a result, dietary interventions aiming to reduce sodium and protein intake may be able to reduce the excretion of osmoles and mitigate polyuria. ${ }^{28}$ The NOCTURNE trial, ${ }^{29}$ which compared the efficacy and safety of a tolvaptan once-daily modified-release formulation with an immediate-release formulation, found no significant difference in daytime and nighttime urine voids between the two modalities. It is also important to note that tolvaptan-induced polyuria was not associated with ureter dilatation after 3 years of treatment, proposing its urologic safety. ${ }^{30}$

\section{Hepatotoxicity}

Drug-induced idiosyncratic liver injury represents a major concern related to tolvaptan therapy and a special warning has been added to its summary of product characteristics. ${ }^{17}$ In the TEMPO 3:4 trial, ${ }^{14}$ tolvaptan-treated patients were at higher risk of presenting important elevations of alanine aminotransferase levels ( $>3$ times the upper normal limit) compared to the placebo group (4.4\% vs $1.0 \%)$. Similar rates of liver injury were noted in the extension TEMPO 4:4 trial $^{15}$ both in the early and delayed-treated participants $(3.8 \%$ and $2.5 \%$, respectively). From this patient population, 3 cases of hepatotoxicity meeting the Hy's Law criteria (defined as elevation of liver function tests $>3$ times the upper normal limit and of bilirubin $>2$ the upper normal limit without cholestasis) were detected; none of them presented acute liver failure and all 3 individuals recovered completely. ${ }^{31}$ It should be stated that in the TEMPO 4:4 trial liver function was evaluated initially every 6 months, subsequently every 3 months and eventually monthly. In contrast, the REPRISE trial ${ }^{16}$ implemented a monthly testing strategy in its entire duration; hence, despite a similar rate of liver function tests' elevation, no severe cases meeting the Hy's Law criteria were detected. To date, one case of acute liver failure necessitating liver transplantation after tolvaptan administration in an ADPKD patient has been described. ${ }^{32}$

Time-to-event analysis of the TEMPO trials indicated that hepatic adverse effects occurred from 3 to 18 months after start of tolvaptan administration, defining thus a window of susceptibility. ${ }^{31}$ This observation was consistent with the open-label extension of all clinical trials, demonstrating that alanine aminotransferase elevations were significantly more common among tolvaptan-naïve patients that were initially treated with placebo in the REPRISE study. ${ }^{26}$ During a median follow-up of 651 days, no Hy's Law cases were detected, suggesting that the effectiveness of the diagnostic strategy of evaluating liver function tests monthly during the first 18 months and every 3 months afterwards. ${ }^{26}$ Regarding drug discontinuation, the outcomes of a small-scale observational study suggested that resumption of tolvaptan was safe in the majority of cases with mild hepatic injury. ${ }^{33}$ It should be noted that a case of hepatotoxicity after the concurrent use of tolvaptan and amoxycillin-clavulanic acid has been recently described, resolving after medication withdrawal. ${ }^{34}$ Interestingly, one of the Hy's Law cases of the TEMPO 3:4 trial occurred after 2 months of an 
amoxycillin-clavulanic acid overdose. ${ }^{31}$ Therefore, further research is warranted to identify any potential synergistic toxicity. Preliminary data regarding the feasibility of tolvaptan administration after liver transplantation come from a report of an ADPKD patient treated with tolvaptan due to progressive kidney disease, without presenting evidence of liver toxicity. ${ }^{35}$

Experimental evidence has proposed that tolvaptan and its metabolites are able to inhibit multiple bile acid transporters, such as MRP2, BSEP and NTCP leading to altered bile homeostasis. ${ }^{36}$ In addition, an ex-situ study in PKC rats, an animal model of ADPKD, demonstrated significantly decreased tolvaptan biliary clearance compared to wild-type rats. ${ }^{37}$ In this context, a study using quantitative systems toxicology modelling indicated that reduced biliary excretion of DM-4103, a tolvaptan's metabolite, may result in bile acid accumulation, depletion of hepatic ATP (adenosine triphosphate) and promote liver injury. ${ }^{38}$ Interestingly, MRP2 expression has been shown to be decreased in PCK rats, a fact that may partially explain the increased susceptibility of ADPKD patients to tolvaptan's cytotoxicity. ${ }^{39}$ Furthermore, in vitro evidence has suggested that liver injury may be mediated by tolvaptan-induced T-cell activation and the subsequent release of specific cytokines, especially interferon- $\gamma$, interleukin-13 and granzyme-B. ${ }^{40}$ Transcriptomic analysis using Collaborative Cross mice showed that tolvaptan may contribute to liver oxidative stress, mitochondrial dysfunction and activation of innate immunity. Interestingly, susceptibility to liver injury correlated with the mRNA expression and plasma levels of secretory leukocyte peptidase inhibitor, indicating its potential role as a predictive factor of hepatotoxicity. ${ }^{41}$

\section{Uric Acid Elevation}

Tolvaptan has been suggested to increase serum uric acid levels by reducing its renal clearance. In the TEMPO 3:4 trial, tolvaptan administration was linked to significantly higher incidence of hyperuricemia (3.9\% vs $1.9 \%$ ) and gout ( $2.9 \%$ vs $1.4 \%$ ) compared to placebo. Nonetheless, no drug discontinuation occurred due to uric acid elevation. ${ }^{14}$ The long-term follow-up of patients included in the TEMPO and REPRISE trials indicated that hyperuricemia was present $2.8 \%$ of the patients receiving tolvaptan. ${ }^{26}$ Regarding the risk of nephrolithiasis, a prospective cohort study showed that tolvaptan was linked to lower supersaturation ratios for calcium oxalate, brushite and uric acid, as well as to reduced urinary net acid excretion, resulting in an enhanced lithogenic $_{\text {profile. }}{ }^{42}$

\section{Muscle Injury}

Two cases of possible tolvaptan-induced elevation of serum creatine kinase have been recently described. The increase was moderate and creatinine kinase returned to normal levels after drug discontinuation. ${ }^{43}$ However, tolvaptan was not readministered to test for recurrence and therefore any potential causal association cannot be currently established.

\section{Discussion}

Tolvaptan has emerged as a promising therapy for patients with ADPKD, able to alter the natural history of the disease by slowing kidney volume increase and glomerular filtration rate decline. Aquaretic events and hepatotoxicity represent its main adverse effects (Figure 1). Specifically, aquaretic adverse effects consist mainly of polyuria, nocturia and polydipsia and may be mitigated by dietary interventions, as well as with the use of urinary osmolality to guide tolvaptan dose titration. On the other hand, druginduced liver injury is typically mild, while severe cases can be effectively prevented by frequent monitoring of liver function tests, especially during the first 18 months. Specifically, the US FDA risk evaluation and mitigation strategy includes the evaluation of serum aminotransferases and bilirubin at 2 and 4 weeks after drug initiation, subsequently monthly during the first 18 months and then every 3 months. The drug is suggested to be promptly discontinued in the presence of laboratory abnormalities or of clinical signs indicative of liver toxicity, such as nausea, anorexia, rash, pruritus, right upper abdominal pain and jaundice. It should be noted that tolvaptan is contraindicated for individuals with history of liver injury, but not in those with uncomplicated polycystic liver disease. ${ }^{13}$ The pathogenetic process of hepatotoxicity is multifactorial, as it is based on the interplay of impaired biliary clearance, activation of innate immunity and increased oxidative stress. Preliminary data about the longterm safety of tolvaptan are available, suggesting that its administration is mainly complicated by polyuria, fatigue and reversible aminotransferase elevations; ${ }^{44}$ however, further real-world research is warranted to draw safe conclusions about is prolonged administration in different clinical settings.

Quality of life has been shown to be significantly impaired in non-dialysis ADPKD patients. $^{45}$ A prospective study has recently evaluated quality of life among ADPKD individuals treated with tolvaptan, 


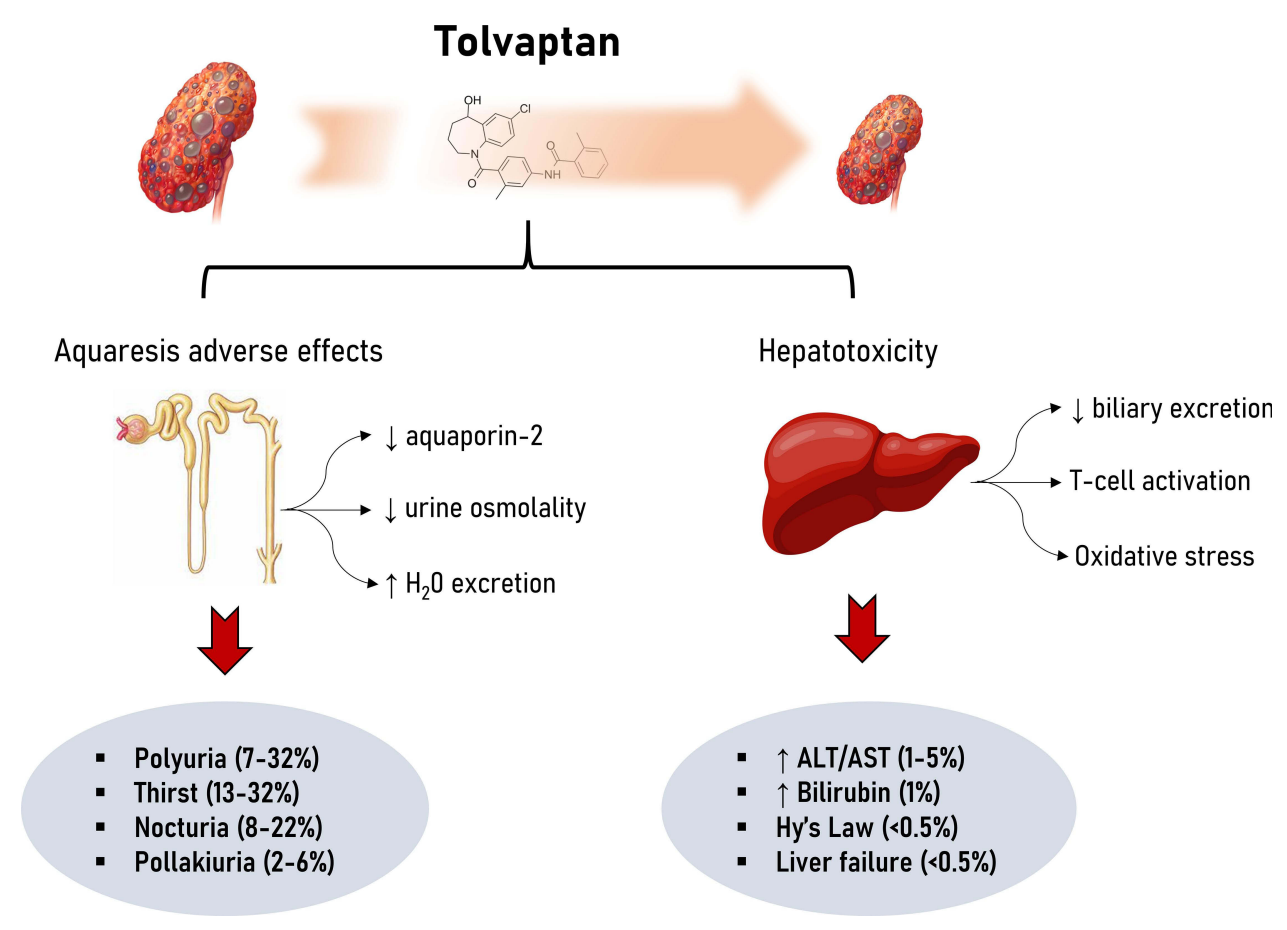

Figure I Tolvaptan adverse effects in patients with autosomal dominant polycystic kidney disease. Tolvaptan administration is mainly associated with aquaretic adverse effects and hepatotoxicity.

Abbreviations: ALT, alanine aminotransferase; AST, aspartate aminotransferase.

indicating that it was not influenced in those tolerating the drug for more than 3 months. Importantly, tolvaptan did not affect cognitive performance, sleep and social support, while it was linked to better patient satisfaction. ${ }^{46}$ However, additional evidence is needed since tolvaptan recipients currently tend to be present higher baseline health status; thus, selection bias cannot be excluded.

Tolvaptan therapy targets to the suppression of vasopressin action in a 24-hour basis. To achieve this, it should be administered in 2 divided doses, 8 hours apart, starting with $45 / 15 \mathrm{mg}$ and up-titrating as tolerated to the maximum dosage of $90 / 30 \mathrm{mg}$ daily. Patients should be advised to ensure appropriate hydration and be encouraged to replenish fluid losses due to nocturia. It is crucial to hold tolvaptan in the setting of acute illness, such as diarrhea, in order to limit the risk of dehydration. ${ }^{24}$ Urinary osmolality and serum sodium may be also routinely monitor aiming to effectively guide tolvaptan dosing. Liver function tests should be measured monthly during the initial 18 months. In case of increased ALT or bilirubin levels, tolvaptan should be temporarily stopped and reinitiated if improvement is observed in repeated tests.

Apart from tolvaptan, several candidate treatments have been suggested for the management of ADPKD patients. The ALADIN trials ${ }^{47,48}$ have proposed that the administration of octreotide long-acting release may delay the progression of renal failure, while its use was linked with non-serious adverse effects, especially diarrhea and cholelithiasis. In contrast, the mTOR inhibitor everolimus with or without octreotide did not lead to beneficial clinical outcomes, but was linked to higher risk of toxicity, such as bone marrow suppression and dyslipidemia. ${ }^{49,50}$ Lanreotide was also ineffective in the DIPAK-1 trial and was associated with gastrointestinal and injection-site side effects. ${ }^{51}$ Regarding the role of metformin, the outcomes of the TAME-PKD trial ${ }^{52}$ are awaited to shed light on its efficacy and the frequency of its adverse effects, especially gastrointestinal ones. Interestingly, lixivaptan has been shown to effectively reduce kidney volume in a rat model of the disease, ${ }^{53}$ while a toxicology modeling study has suggested its beneficial safety profile; ${ }^{54}$ however, its clinical utility remains to be determined.

Future research should confirm the tolvaptan safety profile in large scale by exploiting observational and pharmacovigilance data aiming to provide real-world evidence about the exact long-term incidence and severity of hepatotoxicity and aquaretic adverse effects. In addition, the feasibility of tolvaptan administration in the pediatric population remains to be determined. Specifically, a posthoc analysis of the TEMPO 3:4 trial has proposed the 
efficacy and safety of tolvaptan among young adults ${ }^{55}$; however, data about patients with age $<18$ years is currently sparse. To this end, the outcomes of the NCT02964273 trial are awaited to shed light on tolvaptan therapy in the pediatric population. No data are available concerning tolvaptan in pregnancy; therefore, adequate contraception is suggested for women of childbearing potential. A case of effective tolvaptan administration in a neonate with severe $\mathrm{ADPKD}^{56}$; hence, further evidence is needed to elucidate its role during the neonatal period. Further experimental evidence is also necessary to determine genetic and clinical factors able to predict tolvaptan's toxicity, aiming to optimize patient selection and individualize monitoring, enabling thus a precision medicine approach to ADPKD treatment.

\section{Conclusions}

Tolvaptan administration in patients with autosomal dominant polycystic kidney disease is associated with aquaretic adverse effects and hepatotoxicity risk. Optimal management may promote tolerability and limit drug discontinuations. Future research should confirm the safety profile of tolvaptan in the large-scale setting, elucidate the pathogenetic basis of its toxicity and define its role in specific populations, especially pediatric patients.

\section{Funding}

There is no funding to report.

\section{Disclosure}

The author declares no conflicts of interest.

\section{References}

1. Chapman AB, Devuyst O, Eckardt KU, et al. Autosomal-dominant polycystic kidney disease (ADPKD): executive summary from a Kidney Disease: Improving Global Outcomes (KDIGO) controversies conference. Kidney Int. 2015;88(1):17-27. doi:10.1038/ki.2015.59

2. Suwabe T, Shukoor S, Chamberlain AM, et al. Epidemiology of autosomal dominant polycystic kidney disease in Olmsted county. Clin J Am Soc Nephrol. 2020;15(1):69-79. doi:10.2215/ CJN.05900519

3. Bergmann C, Guay-Woodford LM, Harris PC, Horie S, Peters DJM, Torres VE. Polycystic kidney disease. Nat Rev Dis Prim. 2018;4 (1):1-24. doi:10.1038/s41572-018-0047-y

4. Rastogi A, Ameen KM, Al-Baghdadi M, et al. Autosomal dominant polycystic kidney disease: updated perspectives. Ther Clin Risk Manag. 2019;15:1041-1052. doi:10.2147/TCRM.S196244

5. Bellos I, Kontzoglou K, Perrea DN. Markers of endothelial dysfunction and arterial stiffness in patients with early-stage autosomal dominant polycystic kidney disease: a meta-analysis. Int J Clin Pract. 2021;75(4):e13721. doi:10.1111/ijcp.13721
6. Kuo IY, Chapman AB. Polycystins, ADPKD, and Cardiovascular Disease. Kidney Int Rep. 2020;5(4):396-406. doi:10.1016/j. ekir.2019.12.007

7. Bhatt P, McNeely E, Lin T, Adams K, Patterson J. Review of Tolvaptan's pharmacokinetic and pharmacodynamic properties and drug interactions. J Clin Med. 2014;3(4):1276-1290. doi:10.3390/jcm3041276

8. Li B, Fang D, Qian C, Feng H, Wang Y. The efficacy and safety of tolvaptan in patients with hyponatremia: a meta-analysis of randomized controlled trials. Clin Drug Investig. 2017;37(4):327-342. doi:10.1007/s40261-016-0470-3

9. Luo X, Jin Q, Wu Y. Tolvaptan add-on therapy in patients with acute heart failure: a systematic review and meta-analysis. Pharmacol Res Perspect. 2020;8(3). doi:10.1002/prp2.614

10. Bellos I, Iliopoulos DC, Perrea DN. The role of tolvaptan administration after cardiac surgery: a meta-analysis. $J$ Cardiothorac Vasc Anesth. 2019;33(8):2170-2179. doi:10.1053/j.jvca.2018.12.001

11. Sen J, Chung E, McGill D. Tolvaptan for heart failure in chronic kidney disease patients: a systematic review and meta-analysis. Heart Lung Circ. 2018;27(8):928-939. doi:10.1016/j.hlc.2017.12.013

12. Bellos I, Kontzoglou K, Psyrri A, Pergialiotis V. Tolvaptan response improves overall survival in patients with refractory ascites: a meta-analysis. Dig Dis. 2020;38(4):320-328. doi:10.1159/000503559

13. U.S. FDA. FDA drug safety communication: FDA limits duration and usage of Samsca (tolvaptan) due to possible liver injury leading to organ transplant or death | FDA; 2017. Available from: https:// www.fda.gov/drugs/drug-safety-and-availability/fda-drug-safetycommunication-fda-limits-duration-and-usage-samsca-tolvaptan-duepossible-liver. Accessed June 9, 2021.

14. Torres VE, Chapman AB, Devuyst O, et al. Tolvaptan in patients with autosomal dominant polycystic kidney disease. $N$ Engl $\mathrm{J}$ Med. 2012;367(25):2407-2418. doi:10.1056/NEJMoa1205511

15. Torres VE, Chapman AB, Devuyst O, et al. Multicenter, open-label, extension trial to evaluate the long-term efficacy and safety of early versus delayed treatment with tolvaptan in autosomal dominant polycystic kidney disease: the TEMPO 4:4 trial. Nephrol Dial Transplant. 2018;33(3):477-489. doi:10.1093/ndt/gfx043

16. Torres VE, Chapman AB, Devuyst O, et al. Tolvaptan in later-stage autosomal dominant polycystic kidney disease. $N$ Engl $J$ Med. 2017;377(20):1930-1942. doi:10.1056/NEJMoa1710030

17. FDA. Drug approval package: jynarque (tolvaptan); 2021. Available from: https://www.accessdata.fda.gov/drugsatfda_docs/nda/2018/ 204441Orig1s000TOC.cfm. Accessed April 29, 2021.

18. Torres VE, Wang X, Qian Q, Somlo S, Harris PC, Gattone VH. Effective treatment of an orthologous model of autosomal dominant polycystic kidney disease. Nat Med. 2004;10(4):363-364. doi:10.1038/nm1004

19. Nagao S, Nishii K, Katsuyama M, et al. Increased water intake decreases progression of polycystic kidney disease in the PCK rat. J Am Soc Nephrol. 2006;17(8):2220-2227. doi:10.1681/ASN.2006030251

20. Wang X, Wu Y, Ward CJ, Harris PC, Torres VE. Vasopressin directly regulates cyst growth in polycystic kidney disease. $J$ Am Soc Nephrol. 2008;19(1):102-108. doi:10.1681/ASN.2007060688

21. Gattone VH, Wang X, Harris PC, Torres VE. Inhibition of renal cystic disease development and progression by a vasopressin V2 receptor antagonist. Nat Med. 2003;9(10):1323-1326. doi:10.1038/nm935

22. Reif GA, Yamaguchi T, Nivens E, Fujiki H, Pinto CS, Wallace DP. Tolvaptan inhibits ERK-dependent cell proliferation, $\mathrm{Cl}$ - secretion, and in vitro cyst growth of human ADPKD cells stimulated by vasopressin. Am J Physiol Ren Physiol. 2011;301(5):F1005. doi:10.1152/ajprenal.00243.2011

23. Lee Y, Blount KL, Dai F, et al. Semaphorin 7A in circulating regulatory $\mathrm{T}$ cells is increased in autosomal-dominant polycystic kidney disease and decreases with tolvaptan treatment. Clin Exp Nephrol. 2018;22(4):906-916. doi:10.1007/s10157-018-1542-x

24. Blair HA. Tolvaptan: a review in autosomal dominant polycystic kidney disease. Drugs. 2019;79(3):303-313. doi:10.1007/s40265019-1056-1 
25. Devuyst O, Chapman AB, Shoaf SE, Czerwiec FS, Blais JD. Tolerability of aquaretic-related symptoms following tolvaptan for autosomal dominant polycystic kidney disease: results from TEMPO 3: 4. Kidney Int Rep. 2017;2(6):1132-1140. doi:10.1016/j. ekir.2017.07.004

26. Torres VE, Chapman AB, Devuyst O, et al. Multicenter study of long-term safety of tolvaptan in later-stage autosomal dominant polycystic kidney disease. Clin J Am Soc Nephrol. 2021;16(1):48-58. doi:10.2215/CJN.10250620

27. Kramers BJ, van Gastel MDA, Boertien WE, Meijer E, Gansevoort RT. Determinants of urine volume in ADPKD patients using the vasopressin V2 receptor antagonist tolvaptan. Am J Kidney Dis. 2019;73(3):354-362. doi:10.1053/j.ajkd.2018.09.016

28. Côté G, Asselin-Thompstone L, Mac-Way F, et al. Sodium and urea excretion as determinants of urine output in autosomal dominan polycystic kidney disease patients on V2 receptor antagonists: impact of dietary intervention. Int Urol Nephrol. 2020;52(2):343-349. doi: $10.1007 / \mathrm{s} 11255-020-02384-3$

29. Perrone RD, Chapman AB, Oberdhan D, et al. The NOCTURNE randomized trial comparing 2 tolvaptan formulations. Kidney Int Rep 2020;5(6):801-812. doi:10.1016/j.ekir.2020.03.011

30. Casteleijn NF, Messchendorp AL, Bae KT, et al. Polyuria due to vasopressin V2 receptor antagonism is not associated with increased ureter diameter in ADPKD patients. Clin Exp Nephrol. 2017;21 (3):375-382. doi:10.1007/s10157-016-1297-1

31. Watkins PB, Lewis JH, Kaplowitz N, et al. Clinical pattern of tolvaptan-associated liver injury in subjects with autosomal dominant polycystic kidney disease: analysis of clinical trials database. Drug Saf. 2015;38(11):1103-1113. doi:10.1007/s40264-015-0327-3

32. Endo M, Katayama K, Matsuo H, et al. Role of liver transplantation in tolvaptan-associated acute liver failure. Kidney Int Rep. 2019;4 (11):1653-1657. doi:10.1016/j.ekir.2019.09.002

33. Makabe S, Mochizuki T, Mitobe M, et al. Elevation of the serum liver enzyme levels during tolvaptan treatment in patients with autosomal dominant polycystic kidney disease (ADPKD). Clin Exp Nephrol. 2018;22(5):1079-1087. doi:10.1007/s10157-018-1545-7

34. Pellegrino AM, Annicchiarico Petruzzelli L, Riccio E, Pisani A. Idiosyncratic hepatic toxicity in autosomal dominant polycystic kidney disease (ADPKD) patient in combined treatment with tolvaptan and amoxicillin/clavulanic acid: a case report. BMC Nephrol. 2019;20(1). doi:10.1186/s12882-019-1612-7

35. Uchiyama K, Honda K, Yoshida R, et al. Effect of tolvaptan in a patient with autosomal dominant polycystic kidney disease after living donor liver transplantation. CEN Case Rep. 2016;5 (2):227-231. doi:10.1007/s13730-016-0230-5

36. Slizgi JR, Lu Y, Brouwer KR, et al. Inhibition of human hepatic bile acid transporters by Tolvaptan and metabolites: contributing factors to drug-induced liver injury? Toxicol Sci. 2016;149(1):237-250. doi:10.1093/toxsci/kfv231

37. Beaudoin JJ, Bezençon J, Cao Y, et al. Altered hepatobiliary disposition of tolvaptan and selected tolvaptan metabolites in a rodent model of polycystic kidney disease S. Drug Metab Dispos. 2019;47 (2):155-163. doi:10.1124/dmd.118.083907

38. Beaudoin JJ, Brock WJ, Watkins PB, Brouwer KLR. Quantitative systems toxicology modeling predicts that reduced biliary efflux contributes to tolvaptan hepatotoxicity. Clin Pharmacol Ther. 2021;109(2):433-442. doi:10.1002/cpt.2007

39. Bezençon J, Beaudoin JJ, Ito K, et al. Altered expression and function of hepatic transporters in a rodent model of polycystic kidney disease. Drug Metab Dispos. 2019;47(8):899-906. doi:10.1124/ dmd.119.086785

40. Gibson A, Hammond S, Jaruthamsophon K, Roth S, Mosedale M, Naisbitt DJ. Tolvaptan- and tolvaptan-metabolite-responsive T cells in patients with drug-induced liver injury. Chem Res Toxicol. 2020;33 (11):2745-2748. doi:10.1021/acs.chemrestox.0c00328
41. Mosedale M, Kim Y, Brock WJ, et al. Candidate risk factors and mechanisms for tolvaptan-induced liver injury are identified using a collaborative cross approach. Toxicol Sci. 2017;156(2):kfw269. doi:10.1093/toxsci/kfw269

42. Bargagli M, Dhayat NA, Anderegg M, et al. Urinary lithogenic risk profile in ADPKD patients treated with tolvaptan. Clin J Am Soc Nephrol. 2020;15(7):1007-1014. doi:10.2215/CJN.13861119

43. Agraz-Pamplona I, Larrosa-Garcia M, Bury-Macias RP, Serón-Micas D, Montoro-Ronsano JB. Case report: tolvaptan-associated creatine kinase elevation in two patients with autosomal dominant polycystic kidney disease (ADPKD). Eur J Clin Pharmacol. 2020;76(10):1473-1475. doi:10.1007/s00228-020-02906-z

44. Edwards ME, Chebib FT, Irazabal MV, et al. Long-term administration of tolvaptan in autosomal dominant polycystic kidney disease. Clin J Am Soc Nephrol. 2018;13(8):1153-1161. doi:10.2215/CJN.01520218

45. Neijenhuis MK, Kievit W, Perrone RD, et al. The effect of disease severity markers on quality of life in autosomal dominant polycystic kidney disease: a systematic review, meta-analysis and meta-regression. BMC Nephrol. 2017;18(1). doi:10.1186/s12882-017-0578-6

46. Anderegg MA, Dhayat NA, Sommer G, et al. Quality of life in autosomal dominant polycystic kidney disease patients treated with tolvaptan. Kidney Med. 2020;2(2):162-171. doi:10.1016/j.xkme.2019.11.008

47. Caroli A, Perico N, Perna A, et al. Effect of longacting somatostatin analogue on kidney and cyst growth in autosomal dominant polycystic kidney disease (ALADIN): a randomised, placebo-controlled, multicentre trial. Lancet. 2013;382(9903):1485-1495. doi:10.1016/ S0140-6736(13)61407-5

48. Perico N, Ruggenenti P, Perna A, et al. Octreotide-LAR in later-stage autosomal dominant polycystic kidney disease (ALADIN 2): a randomized, double-blind, placebo-controlled, multicenter trial. PLoS Med. 2019;16(4):e1002777. doi:10.1371/journal.pmed.1002777

49. Walz G, Budde K, Mannaa M, et al. Everolimus in patients with autosomal dominant polycystic kidney disease. $N$ Engl J Med. 2010;363(9):830-840. doi:10.1056/NEJMoa1003491

50. Chrispijn M, Gevers TJG, Hol JC, Monshouwer R, Dekker HM, Drenth JPH. Everolimus does not further reduce polycystic liver volume when added to long acting octreotide: results from a randomized controlled trial. J Hepatol. 2013;59(1):153-159. doi:10.1016/j.jhep.2013.03.004

51. Meijer E, Visser FW, Van Aerts RMM, et al. Effect of lanreotide on kidney function in patients with autosomal dominant polycystic kidney disease the DIPAK 1 randomized clinical trial. JAMA. 2018;320 (19):2010-2019. doi:10.1001/jama.2018.15870

52. Seliger SL, Watnick T, Althouse AD, et al. Baseline characteristics and patient-reported outcomes of ADPKD patients in the multicenter TAME-PKD clinical trial. Kidney360. 2020;1(12):1363-1372. doi:10.34067/kid.0004002020

53. Wang X, Constans MM, Chebib FT, Torres VE, Pellegrini L. Effect of a vasopressin V2 receptor antagonist on polycystic kidney disease development in a rat model. Am J Nephrol. 2019;49(6):487-493. doi:10.1159/000500667

54. Woodhead JL, Pellegrini L, Shoda LKM, Howell BA. Comparison of the hepatotoxic potential of two treatments for autosomal-dominant polycystic kidney disease using quantitative systems toxicology modeling. Pharm Res. 2020;37(2). doi:10.1007/s11095-019-2726-0

55. Raina R, Chakraborty R, DeCoy ME, Kline T. Autosomal-dominant polycystic kidney disease: tolvaptan use in adolescents and young adults with rapid progression. Pediatr Res. 2021;89(4):894-899. doi:10.1038/s41390-020-0942-2

56. Gilbert RD, Evans H, Olalekan K, Nagra A, Haq MR, Griffiths M. Tolvaptan treatment for severe neonatal autosomal-dominant polycystic kidney disease. Pediatr Nephrol. 2017;32(5):893-896. doi:10.1007/s00467-017-3584-9 


\section{Publish your work in this journal}

Therapeutics and Clinical Risk Management is an international, peerreviewed journal of clinical therapeutics and risk management, focusing on concise rapid reporting of clinical studies in all therapeutic areas, outcomes, safety, and programs for the effective, safe, and sustained use of medicines. This journal is indexed on PubMed Central, CAS,
EMBase, Scopus and the Elsevier Bibliographic databases. The manuscript management system is completely online and includes a very quick and fair peer-review system, which is all easy to use Visit http://www.dovepress.com/testimonials.php to read real quotes from published authors.

Submit your manuscript here: https://www.dovepress.com/therapeutics-and-clinical-risk-management-journal 